\title{
Seeking to introduce a pharmacoeconomics framework in Cyprus
}

\author{
Panagiotis Petrou ${ }^{1}$
}

Received: 8 December 2017 / Revised: 30 July 2018 / Accepted: 15 September 2018/Published online: 3 October 2018

(C) Swiss School of Public Health (SSPH+) 2018

Health agencies face an array of profound challenges. Among them, the need to exert control over surging pharmaceutical expenditures is imperative (Dubois et al. 2000). If pharmaceutical expenditure is left unchecked, it threatens to jeopardise health systems' fiscal sustainability and violate equity and solidarity, which are fundamental principles in health care provision. Pharmaceutical care has significantly contributed towards life's expectancy increase and morbidity reduction, so health agencies must balance the need to keep affordable prices and give patients access to innovative products (Lichtenberg 2012).

Usually, the price of a pharmaceutical is nested in its intellectual property rather than in its value or its ingredients. The asymmetry of information between involving parties deprives patients from the ability to make informed decision-making and blurs the transmission of pharmaceutical's price as a signal. This leaves countries embroiled in significant troubles while trying to satisfy the discordant needs of patients, physicians but also abide by the national fiscal policies as well.

High prices constitute a major cause of delay in the reimbursement process. This delay impedes timely access, a fundamental function of a health system, which comprises a decisive factor in many debilitating and degenerative diseases. Apart from the potential negative impact on health, this lingering may expose patients to potentially catastrophic out-of-pocket expenditures, if they opt to buy an indicated medicine out-of-pocket.

Of particular interest is the fact that the prices of pharmaceutical products do not correlate to their value. Value is a highly unmeasured and misinterpreted term. Yet, even within the limitations of its conceptual vagueness, value, as attested by hard clinical endpoints, should constitute the

This Editorial is part of the series "Young Researcher Editorial", a training project of the Swiss School of Public Health.

\footnotetext{
Panagiotis Petrou

panayiotis.petrou@st.ouc.ac.cy

1 HIO, Nicosia, Cyprus
}

core of any health system. And value is rather rare in the pharmaceutical sector (Kantarjian et al. 2013).

To this direction, the goal of our study was to develop a pricing scheme which provides that the price of a pharmaceutical is set pertinent to its value and related direct and indirect costs, in the form of a value-based pricing (VBP) scheme. Specifically, we set pharmaceutical prices based on their incremental (compared to the current therapeutic benchmark) medical, social and financial benefits and costs, in the context of a defined willingness- to-pay (WTP) threshold. The defined value-based (VB) price comprises not only a signal, but it also embeds the acceptable health utility that it will be foregone in the broader health system. We also explored the conceptual and operational framework of the VBP. This includes practical ways to implement the VBP, assessment of its limitations and ways to extenuate their impact.

Our study focused on oncology, which accounts for up to $30 \%$ of total health expenditures. The increase rate of oncology products is greater than of all other corresponding sectors, increasing $100 \%$ between 2005 and 2014. We further focused on metastatic renal cell carcinoma, a rare cancer, with limited therapeutic options. We developed a Markov Model which simulates disease progression and included all relevant costs and health gains. The output of the model was the definition of the VB price of sorafenib to best supportive care (BSC). This VB price rendered the product cost-effective based on WTP thresholds suggested by the WHO (Dias et al. 2013; Petrou and Talias 2014). In addition, this VB price captured all medical and societal gains, including survival, disease progression, quality of life, side effects and all direct and indirect costs. We concluded that the VB price of sorafenib should have been $28 \%$ lower than its current retail price. In Cyprus, pharmaceutical prices are set at the European average. Since our model was mainly sensitive to efficacy data mined from international studies, and depended much less on local data, our results could serve as a proxy for other countries (Petrou and Talias 2013).

VBP comprises a paradigm shift that distributes risk among payer and industry and is evocative of delivering 
tangible value to payers through the price, which acquires a binary attribute: signal and gauge for reimbursement. VBP can also give patients quicker access to new products, which is crucial for life-threatening conditions where survival expectancy is low. Furthermore, VBP can fairly incentivise the industry and reward genuine innovation, factors that are desirable for the Industry.

My study highlighted some unsolved aspects of VBP. Interest in VBP in the US, UK, Germany and Sweden, and Italy's experience with risk sharing schemes, may pave the way to address these issues (Flume et al. 2016). VBP must be scalable and applicable to all pharmaceutical products. Data on efficacy should be cautiously gathered. A consensus should be drafted by stakeholders on whether efficacy or real-life effectiveness data are more important. We should also underline that the recent advances in statistical modelling should make it easier to implement VBP.

VPB would help us initiate a new, holistic, rational and comprehensive era of disease management that will cast light to the confluence of costs and meaningful medical outcomes.

\section{Compliance with ethical standards}

Conflict of interest I have not received funding for this research in any form, including travel grants, fellowship, commercial assistance, equipment, honoraria or other payments that may constitute a conflict of interest.

Ethical approval This study was not performed on humans.

\section{References}

Dias S, Welton NJ, Sutton AJ et al (2013) Evidence synthesis for decision making 1: introduction. Med Decis Mak 33(5):597-606

Dubois RW, Chawla AJ, Neslusan CA et al (2000) Explaining drug spending trends: does perception match reality? Health Aff 19(2):231-239

Flume M, Bardou M, Capri S et al (2016) Feasibility and attractiveness of indication value-based pricing in key EU countries. J Mark Access Health Policy 4(1):1-6

Kantarjian M, Fojo T, Mathisen M et al (2013) Cancer drugs in the United States: Justum Pretium-the just price. J Clin Oncol 31(28):3600-3604

Lichtenberg FR (2012) The contribution of pharmaceutical innovation to longevity growth in Germany and France, 2001-2007. PharmacoEconomics 30(3):197-221

Petrou P, Talias M (2013) A framework for applying health technology assessment in Cyprus: thoughts, success stories, and recommendation. Value Health Reg Issues 2:273-278

Petrou P, Talias M (2014) A pilot study to assess feasibility of value based pricing in Cyprus through pharmacoeconomic modelling and assessment of its operational framework: sorafenib for second line renal cell cancer. Cost Eff Resour Alloc 12(12):1-11 